\section{Septic Polyarthritis Caused by Streptobacillus moniliformis}

\author{
Ali Uddin, Tung Phan, Mohamed Yassin \\ Author affiliation: University of Pittsburgh, Pittsburgh, \\ Pennsylvania, USA
}

\section{DOI: https://doi.org/10.3201/eid2712.210649}

Streptobacillus moniliformis is a pleomorphic, fastidious gram-negative bacillus that colonizes rodent respiratory tracts and causes rat-bite fever in humans. Rat-bite fever is associated with septic arthritis, usually monoarticular or pauciarticular. We report a rare case of polyarticular septic arthritis caused by S. moniliformis; the disease was initially misdiagnosed as inflammatory arthritis.

Streptobacillus moniliformis is a pleomorphic, fastidi$\mathcal{S o u s}$ gram-negative bacillus commonly found in the nasopharynxes of rats and other rodents (1). It is transmitted to humans through rat bites, scratches, or ingestion of food or water contaminated with rat feces (2), as exemplified by a 1926 outbreak in Haverhill, Massachusetts, USA (3). Symptoms usually comprise fever, headache, pharyngitis, myalgia, migratory arthralgia, and vomiting, followed by a maculopapular rash on extensor surfaces. Arthralgia related to reactive polyarthritis develops in $\approx 50 \%$ of patients (4). Wang et al. (5) reported a well-documented case of septic arthritis and reviewed 11 cases in the literature; 5 of 12 cases had local signs of arthritis but not fever or general sepsis (5).

In January 2021, a 59-year-old cleaning woman sought treatment for 3 consecutive days at the emergency department before she was admitted for 2 months of progressively worsening left knee pain. She did not have a rash or fever. Her medical history included ovarian cancer, which was treated surgically 20 years before, and cervical stenosis after C2-T1 fusion. Radiographs of her knee showed mild arthritis; arthrocentesis conducted at the first emergency department visit produced synovial fluid with no organisms visible by Gram staining. The patient was prescribed steroids for inflammatory arthritis, but joint pain and swelling did not improve. At her third visit to the emergency department, she was afebrile and had tender, warm, and swollen knees, wrists, right shoulder, and left ankle; these joints also showed a decreased range of motion (Figure). She did not have a rash or lymphadenopathy. Seven days after admission, a second arthrocentesis produced synovial fluid with 40,000 leukocytes/ $\mathrm{mL}^{3}$, no organisms visible by Gram staining, and no crystals. Other rheumatic results were within reference ranges. On
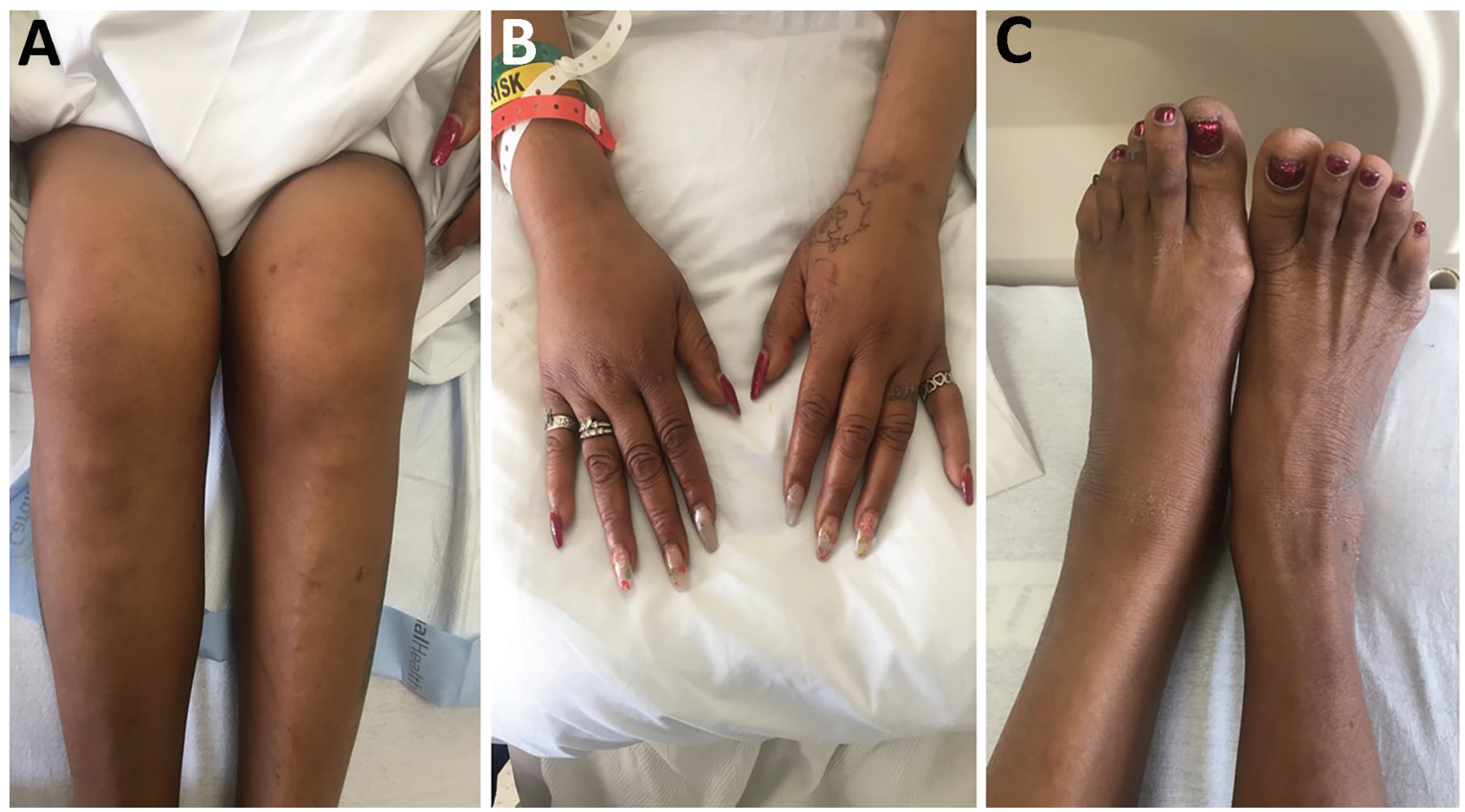

Figure. Tender, warm, and swollen knees (A), wrists (B), and left ankle (C) with decreased range of motion in a patient with septic polyarthritis caused by Streptobacillus moniliformis infection, United States. 
day 11, tiny colonies grew poorly on sheep blood agar (Appendix Figure, https://wwwnc.cdc.gov/ EID/article/27/12/21-0649-App1.pdf). We did not observe growth on chocolate, MacConkey, or Columbia colistin-nalidixic acid agars. Microscopic examination of a Gram-stained smear revealed gram-negative rods with bulbar swellings (Appendix Figure). We used matrix-assisted laser desorption/ionization time-of-flight mass spectrometry to confirm the colonies as S. moniliformis (score 2.35); we did not conduct susceptibility testing.

We diagnosed subacute polyarticular septic arthritis, which has a recommended treatment of penicillin $G(200,000$ units $2 \times / d$ for $5-7$ days); the alternative option is a 4 -week course of ceftriaxone. We stopped steroid treatment and prescribed ceftriaxone because the patient had a severe penicillin allergy. She responded very well to intravenous treatment, and her joint pain and swelling improved remarkably. Two months before symptom onset, she had cleaned a research laboratory housing rats and homes that had mousetraps. She was not aware of any bites or scratches. We obtained informed consent for her participation in this research.

S. moniliformis is the etiologic agent of rat-bite fever, which usually causes fever, rash, and arthralgia. However, this patient and others had polyarticular involvement without fever or rash $(5,6)$. Previous case reports have described $S$. moniliformis as favoring synovial and serosal surfaces $(7,8)$.

S. moniliformis is difficult to identify because of its fastidious nature and slow growth on culture; as a result, it is sometimes misdiagnosed as inflammatory arthritis. An informed diagnosis requires raised clinical awareness and attention to patient social history. Arthrocentesis should be conducted in any case of suspected septic arthritis. As shown in this case, matrix-assisted laser desorption/ionization time-offlight mass spectrometry is a useful tool for diagnosing S. moniliformis infection.

\section{Acknowledgment}

We thank the staff of the clinical microbiology laboratory at UPMC Mercy for help with initial isolation and characterization of the isolate.

\section{About the Author}

Dr. Uddin is a resident in internal medicine at the University of Pittsburgh, Pittsburgh, Pennsylvania, USA. Her research interests include infectious disease.

\section{References}

1. Strangeways WI. Rats and carriers of Streptobacillus moniliformis. J Pathol Bacteriol. 1933;37:45-51. https://doi.org/ 10.1002/ path.1700370106

2. Centers for Disease Control and Prevention. Fatal rat-bite fever - Florida and Washington, 2003. MMWR Morb Mortal Wkly Rep. 2005;53:1198-202.

3. Place EH, Sutton LE. Erythema arthriticum epidemicum (Haverhill fever). Arch Intern Med (Chic). 1934;54:659-84. https://doi.org/10.1001/archinte.1934.00160170002001

4. Stehle P, Dubuis O, So A, Dudler J. Rat bite fever without fever. Ann Rheum Dis. 2003;62:894-6. https://doi.org/10.1136/ard.62.9.894

5. Wang TK, Wong SS. Streptobacillus moniliformis septic arthritis: a clinical entity distinct from rat-bite fever? BMC Infect Dis. 2007;7:56. https:// doi.org/10.1186/1471-2334-7-56

6. Dendle C, Woolley IJ, Korman TM. Rat-bite fever septic arthritis: illustrative case and literature review. Eur J Clin Microbiol Infect Dis. 2006;25:791-7. https:/ / doi.org/10.1007/ s10096-006-0224-x

7. Rumley RL, Patrone NA, White L. Rat-bite fever as a cause of septic arthritis: a diagnostic dilemma. Ann Rheum Dis. 1987;46:793-5. https://doi.org/10.1136/ard.46.10.793

8. Adams SH, Mahapatra R. Rat bite fever with osteomyelitis and discitis: case report and literature review. BMC Infect Dis. 2021;21:479-86. https:// doi.org/10.1186/ s12879-021-06172-x

Address for correspondence: Mohamed Yassin, Division of Infectious Diseases, University of Pittsburgh, Pittsburgh, PA 15219, USA; email: yassinm@upmc.edu

\section{Coxiella burnetii in 3 Species of Turtles in the Upper Midwest, United States}

William E. Sander, Richard King, William Graser, Joshua M. Kapfer, Aubrey I. Engel, Laura Adamovicz, Matthew C. Allender

Author affiliations: University of Illinois Urbana-Champaign, Urbana, Illinois, USA (W. E. Sander, A.I. Engel, L. Adamovicz, M.C. Allender); Northern Illinois University, DeKalb, Illinois, USA (R. King); Kane County Forest Preserve, Geneva, Illinois, USA (W. Graser); University of Wisconsin-Whitewater, Whitewater, Wisconsin, USA (J.M. Kapfer)

DOI: https://10.3201/eid/2712.211278 